\title{
The social production of evidence: regenerative medicine and the 21 st Century Cures Act
}

\author{
Linda F Hogle*,1 \& Amritava Das² \\ ${ }^{1}$ Department of Medical History \& Bioethics, Stem Cell \& Regenerative Medicine Center, University of Wisconsin-Madison, \\ Madison, WI, USA \\ ${ }^{2}$ Wisconsin Institute for Discovery, University of Wisconsin-Madison, Madison, WI, USA \\ * Author for correspondence: Ifhogle@wisc.edu
}

First draft submitted: 14 April 2017; Accepted for publication: 1 August 2017; Published online: 4 October 2017

Keywords: 21 st Century Cures Act $\bullet$ clinical trials $\bullet$ policy $\bullet$ regulatory review $\bullet$ Right-to-Try laws $\bullet$ stem cells $\bullet$ US

Regenerative medicine (RM) has experienced uncertainty in regulatory review since the beginning. Political and social environments always affect policy: consistency and interoperability across societies has been hard to achieve, and over time, extreme precaution has largely been replaced with optimism and high expectations from patients, politicians and investors. Like all novel therapies, disputes about what sort of evidence matters and how it should best be produced dogged the field's early days. With little expertise from regulators or industry, and few standards to go on, safety and effectiveness requirements were negotiated. More recently, assumptions about what counts as acceptable evidence are being up-ended in light of new tools and techniques for the collection and analysis of data, but also decades of discontent with evidence-based medicine (EBM). Patients are also demanding to have a say in clinical research and may be willing to take greater risks. All this is happening in a political climate in which funding is being cut for scientific research and governmental oversight.

Recent US laws portend further changes. While intended to provide a clear and lubricated pathway for product approval, new uncertainties may arise for RM. The US 21st Century Cures Act [1] (hereafter 'Cures Act') makes statutory changes to the US Department of Health and Human Services (DHHS) and the US FDA, directing the agencies to expedite product approvals by removing requirements viewed as burdensome while considering alternative forms of evidence. This entails fundamental changes to regulatory frameworks altering how evidence may be collected and evaluated. Simultaneously, Right-To-Try (RTT) laws aim to broaden access to experimental treatments with only preliminary evidence.

Commentators portray the changes on the one hand as liberating patient access to treatments, encouraging innovation and lowering regulatory burdens, and on the other, encouraging false hope among vulnerable patients, attacks on science, lobbyist-driven maneuvering, and lowering rigorous standards. However, framing such controversies over regulatory review as polarities oversimplifies the situation. We argue that current changes need to be viewed within the broader contexts of social phenomena that shape what comes to count as valid evidence. We first briefly highlight features of the Cures Act, then situate it historically and politically within the longer history and broader contexts of evidence production. We close with implications for RM.

\section{New paradigms for producing evidence?}

The Cures Act was billed as a patient-centered effort to promote innovative therapies and reduce activities seen as barriers to research. One of the few successful bipartisan legislative efforts in a divisive Congress, it passed overwhelmingly at the end of 2016. This broad-sweeping law has many components, including increasing funding for specific medical research areas, authorizing broader data sharing, and reducing bureaucratic processes. Significantly, it directs the FDA to consider new forms of evidence and creates new mechanisms for accelerated review for RM products.

Notably, a section entitled 'Modern Trial Design and Evidence Development' [2] directs the FDA to consider forms of evidence other than those produced by canonical EBM methods, especially randomized clinical trials 
(RCTs). In particular, observational data, such as clinical outcome assessment (defined as "measurement of a patient's symptoms, overall mental state, or the effects of a disease or condition on how the patient functions"), case histories, data mining of registries or medical records, surveys and even non peer-reviewed journal articles may be submitted for both pre- and postmarket evaluations. Title IIIA [3] codifies the use of 'patient experience data' (including "data collected by patients, parents, caregivers, patient advocacy organizations, disease research foundations, researchers, and drug manufacturers") concerning the impact of the therapy on patients' lives. Primarily for risk-benefit analyses, such data can be collected throughout the review. Other patient-generated data may come from textual narratives in social media or medical histories, or from mobile health devices (smartphone apps, sensors or telemetry on dedicated devices). Such data are intended to represent patients' 'real-world' experience. Real world evidence (RWE) is defined in the law as "data regarding the usage, potential benefits or risks, of a drug derived from sources other than randomized clinical trials" [4]. RWE is allowed in both pre- and post-market phases of approval.

Significantly, Section 3033 creates a new designation of products as 'Regenerative Advanced Therapies' (RAT) [5] (Regenerative Medicine Advanced Therapies [RMAT] is the FDA designation [6] for technologies covered by Section 3033 [5] RAT of the Cures Act). While a request for the designation must accompany an Investigational New Drug (IND) application, it allows expedited review of products in cases of; "serious or life-threatening conditions, and preliminary evidence indicates that it has potential to address unmet needs. "The sponsor of a RMAT-designated product may also fulfill any postapproval requirements by submitting "clinical evidence, clinical studies, patient registries, or other sources of real world evidence, such as electronic health records" or by monitoring treated patients.

The law also promotes the use of tools and techniques advocated in previous policy documents shifting from 'evidence-based' to 'value-based' assessments (which also take outcomes and cost-effectiveness into account) [7]. This includes expanded use of biomarkers and surrogate end points, and new data tools. While biomarkers and surrogate end points can be useful measures of efficacy in some cases and may or may not be of clinical importance by themselves, they may not indicate that the patient's condition has actually improved, and in some cases, focusing on biomarkers has meant that other risks or comorbidities are missed. For example, the drug Avandia lowered $\mathrm{HbA}_{1 \mathrm{c}}$ in diabetic patients, but increased their rate of heart attacks. While approved for use as evidence since 1992 , the use of biomarkers still raise questions about clinical relevance [8].

The Cures Act further instructs the FDA to use adaptive clinical trial designs, Bayesian probability methods and predictive analytics consistent with the expanding uptake of big data analytics and other computational studies. Under the new regulations, preclinical studies (always a challenge for RM [8]) may be augmented with computational studies. While comparatively inexpensive and able to handle large volumes of very complex data, the problems and limitations of associative analyses using big data, are well-recognized [9]. When used to make sense of RWE - as is being done in contemporary studies to predict patient social and behavioral patterns and predict outcomes as other forms of real-world evidence - associative techniques are highly problematic.

The emphasis on safety and apparent reduction in rigorous requirements to demonstrate efficacy is consistent with the growing number of state RTT laws, which allow for the use of experimental treatments for patients with life-threatening illnesses or those addressing 'unmet needs' however vaguely defined. A federal-level law has also been proposed (Trickett-Wendler Right-To-Try Act [10]). Such laws propose to accelerate access by removing requirements to go through rigorous, larger-scale Phase III clinical trials [11]. Similarly, the proposed Reliable and Effective Growth for Regenerative health Options that improve Wellness (REGROW) Act [12], aimed to lessen requirements specifically for experimental stem cell treatments. While never enacted, much of the language and intent was incorporated into the Cures Act, particularly in the description of Regenerative Advanced Therapies. Questions remain about whether clinics will try to use this language to circumvent FDA guidance regarding homologous use and minimal manipulation [13].

\section{Expertise, experience \& expediency}

The alternative forms of data as proposed in the Cures Act represent a sea-change in the way of thinking about evidence. Observational data have been used to evaluate and adjust experimental treatments, but because they are more subject to bias, have been considered as less reliable than controlled experimental conditions of EBM [14,15]. 'Gold-standard' RCTs have instead dominated thinking about EBM for decades [16-18]. A social movement of methodological reformers in the second half of the 20th century pushed for controlled conditions with hypothesisdriven, experimental design, arguing that a more systematic approach would enable generalization of findings across populations and settings, and distance investigators from subjects (and from product companies) [19]. Formalized, elite expertise was intended to avoid precisely the kinds of bias and spurious relationships of observational data that 
now appears in the new law. Systematic methods also make it easier to replicate findings, avoiding waste of research funding and resources [20].

Nevertheless, criticisms of EBM abound. In fact, Rosemann and Chaisinthop argue that resistance to EBM is becoming more organized and global, especially in stem cell medicine [21]. RCTs have been criticized as too costly, lengthy and burdensome, hence increasingly less feasible to execute [22]. Public health professionals argue that EBM disregards social determinants of health. Social and economic conditions make a great deal of difference in treatment processes and outcomes, but the highly controlled protocols required for participation in a RCT create artificial conditions and do not reflect the way people actually live and the conditions in which they would receive the treatment if approved. Controlled studies use narrowly defined research questions with data collection and analyses that may miss phenomena and unanticipated reactions that may occur during the course of the trial, including changes in the lived experiences of subjects. As Löwy notes, human bodies are major instruments of the development and validation of medical knowledge, yet they are not passive experimental objects [23]. Social scientists and bioethicists have long argued that recruiting highly selected, homogenous groups from which to generalize about whole populations is problematic: diversity in terms of sex, age, geography and history matters [24].

Deciding what comes to count as evidence is profoundly political, and certainly not new. Waves of discontent about evidence requirements and methodology resulted in reforms in 1938 and the 1960s [19]. In the 1990s, politicians and industry representatives railed against regulatory burdens and slow-downs as a part of broader 'downsizing government' initiatives, a theme also running through the 2016 US presidential election. For some 15 years, the US Institute of Medicine has recommended alternatives to EBM, recommending in silico trials, adaptive trial designs, and allowing for 'evolving evidence', including patient-generated data, largely for cost efficiency $[7,25]$. In combination with resurgent debates about allowing access to experimental treatments and everpresent calls for accelerating translation of products into clinical use, criticisms of EBM fueled proposals for new laws and regulatory policy to alter what is considered as acceptable evidence. That includes the FDA's Regulatory Science Initiative [26] to allow more adaptive trial designs, greater use of biomarkers and surrogate end points, and new tools and techniques (especially computational modeling and simulations) to augment submission portfolios, especially for novel therapies.

Regulatory processes are thus a site for the negotiation of credibility, authority and the determination of what kinds of expertise are seen to be both relevant and expedient. Still, there are real-world issues to deal with when modifying the way evidence is produced.

\section{Real-world problems of 'real-world evidence' in regenerative medicine}

First, there is a host of technical and pragmatic challenges with RM which makes evidence production both more complicated and more critical to get right. RM is intrinsically different from other clinical products, due to the complexity of mechanisms and as-yet unknown long-term effects in human hosts. There is also more at stake because as first-in-human research, RM studies bear high risk with potentially little benefit. Proof of safety is critical, but there should also be careful preclinical research and good indication of efficacy to justify trials in humans [27].

Many RM products are combination products or the mechanism(s) of action depend on more than one active component, making it difficult to meet the FDA's definition of potency. Cells integrate into tissues, and tissues remodel naturally over time, so knowing if changes resulted from the inserted cells, some effect exerted on endogenous processes (such as paracrine signaling or cytokine release), or other native physiological mechanisms unrelated to the stem cells is difficult. Changes may take some time to appear, so may be missed in preclinical data with the short lifespan of small animals, and in humans without long-term follow-up. End points are also difficult to determine in cell-based therapies. How much cell survival and engraftment is clinically significant? Which patient-reported responses measure meaningful benefit? Cells can migrate and stimulate cascades of signals affecting tissues other than intended targets. Yet in very ill patients, how can effects of comorbidities be discerned from off-target effects?

This points to the second problem; effectiveness and clinical outcomes as measured by regulatory requirements may differ significantly from what patients experience as relevant. The FDA defines 'potency' as "the specific ability of the product, as indicated by appropriate laboratory tests or by adequately controlled clinical data obtained through the administration of the product in the manner intended, to effect a given result" (21 CFR 600.3 (s)) [28]. But while there may be a correlation of potency and clinical effectiveness by regulatory definitions, direct cause and effect is hard to prove, and may not match patient-perceived effectiveness as reflected in reports of patient experience. 
Related are pragmatic problems: how should RWE best be collected and analyzed? Can it be generalized? Validated? Will small firms have the wherewithal to collect such data, or will the costs be more than offset by savings from not being required to recruit and conduct large-scale trials if their product receives a RMAT designation? There is also considerable ambiguity about terminology, such as what conditions might qualify as an 'unmet need' and how broadly RWE will be defined.

Fourth, there is an odd dilemma for social scientists and bioethicists who have for years agitated to take patients' social situatedness and context into account [24]. It is important to take seriously what matters most to those who are suffering, yet elevating anecdotal narratives from caregivers, family members and patients in the evidence hierarchy comes precariously close to testimonials used in lieu of evidence by clinics offering experimental treatments outside of trials. Bioethicists have criticized this as exploitive [13,29]. This raises the politics of risk and participation: who bears what kind of risk, and under what conditions? The new legislation occurs in the wake of controversial approvals of products and treatments in response to intense patient and political pressure despite little or no evidence of effectiveness [13,29]. Challenges to the credibility of data sources also resurrect old debates about lay versus elite expertise. Patient activists increasingly matter as interlocutors in clinical research and the negotiation of credibility of authority and expertise [24,30]. Yet while the law institutionalizes more direct extraction of patients' experience, there is no guarantee that they will have a say in how it will be used or how the process will be governed.

Finally, regulatory review does not occur in a vacuum; it interdigitates with other laws and political-economic exigencies. It remains to be seen how the apparent shift away from more rigorous proofs of efficacy may conflict with fundamental features of the Affordable Care Act. This major healthcare reform law shifts payment systems from fee-for-service to a value-based system, whereby reimbursement is based on treatments with documented improved clinical outcomes and enhanced cost efficiency as defined by measures in the law [25]. Having less conventional evidence typically makes it less likely that payers will approve experimental treatments for reimbursement, but perhaps that will change. 'Patient-generated' data are used along with conventional clinical measures, but is defined and used differently. How will various forms of alternative evidence be operationalized in differing healthcare contexts?

The Cures Act may meet unanticipated hurdles in the new US presidential administration. Executing the new mandates requires new guidelines and regulations, but new executive orders require federal agencies to eliminate two prior regulations for every new regulation issued, and cap costs on regulations (incremental cost of all new regulations should be no greater than net zero). Which regulations affecting issues of public health and safety should be eliminated, and through what process? Taxpayer-funded agencies have also been discouraged from communicating to the public (which includes scientific researchers, economic analysts, journalists, scientific integrity experts and the general public), constraining public debate. Finally, some provisions may remain as unfunded mandates, since insufficient funding exists to develop the sort of data infrastructures and procedures necessary to accomplish the aims. Nevertheless, the Act requires performance measures with audit reporting.

\section{Discussion}

Processes of producing evidence are never static and always contested. Research governance has always been as much about power and persuasion as about matters of fact [17]. More than 1400 lobbyists from pharmaceutical and device companies and patient advocacy groups weighed in on the Cures Act, and activists of all stripes continue to be engaged in state and federal-level policy debates. In that sense, what is of interest regarding the new laws is not simply whether or not an evidence bar is lowered; as historians have shown, bars have been and will continue to be raised and lowered. Rather, it is important to consider the form that evidence takes and what (whose) expertise and experience comes to count, and how expediency should be balanced with rigor.

The Cures Act and RTT acts provide the institutional means to elevate alternative sources of information, but doing so disturbs long-held beliefs about what comes to count as credible evidence as well as the processes through which it is produced. Augmenting or substituting alternative forms of evidence shortens the time and cost of studies, lessens demands on product sponsors, and perhaps speeds innovations to patients, but may reduce the scientific rigor of the trials being conducted. There are valid reasons to rethink conventional trials for RM, including the incorporation of lived experiences of patients. Yet weighting real-world evidence in the way put forward in the Cures Act and in some in RTT Laws is concerning. The new laws draw attention to the fact that the modes of governing the production of evidence are inherently social: tools and techniques, new methods of collecting and assessing data, new participants and power dynamics affect what comes to count as relevant evidence. 
Aspirants may flood the FDA with RMAT applications, hoping to be subject to less scrutiny or a lowered bar. We have confidence that the FDA now has the expertise to evaluate substantive data presented by RM product sponsors and will not simply green-light RM products with insufficient documentation of safety and efficacy. We also have confidence that responsible RM leaders will continue to maintain high ethical standards and develop good practice as new issues arise. We do worry about the prevailing political environments that may insufficiently fund regulatory review expertise and crucial long-term postapproval analysis. Evidential 'bars' will be adjusted as the new laws are implemented, but we underscore that damage done now to the burgeoning field as a result of inadequate or inappropriate data will have long-term effects on the field. As RM investigators gain experience and expertise, we are hopeful that they will carefully consider what counts as appropriate, not just expedient evidence.

\section{Financial \& competing interests disclosure}

The authors have no relevant affiliations or financial involvement with any organization or entity with a financial interest in or financial conflict with the subject matter or materials discussed in the manuscript. This includes employment, consultancies, honoraria, stock ownership or options, expert testimony, grants or patents received or pending, or royalties.

No writing assistance was utilized in the production of this manuscript.

\section{References}

1. 21st Century Cures Act 2016, Public Law 114-255.

2. Ibid. $\S \S 3021-3024$.

3. Ibid. $\S \S 3001-3004$.

4. Ibid. $\S \S 3022$.

5. Ibid. $\S \S 3033$.

6. US FDA. Regenerative medicine advanced therapy designation (2017). www.fda.gov/BiologicsBloodVaccines/CellularGeneTherapyProducts/ucm537670.htm

7. Institute of Medicine. The Learning Healthcare System. Olsen LA, Aisner D, McGinnis JM (Eds). National Academies Press, DC, USA (2007).

8. Barazzetti G, Hurst SA, Mauron A. Adapting preclinical benchmarks for first-in-human trials of human embryonic stem cell-based therapies. Stem Cells Transl. Med. 5(8), 1058-1066 (2016).

9. Hoffman S, Podgurski A. The use and misuse of biomedical data: is bigger really better? Am. J. Law Med. 39(4), 497-538 (2013).

10. Trickett Wendler Right-To-Try Act of 2017, S. 204, 115th Cong. (2017).

11. Bateman-House A, Kimberly L, Redman B, Dubler N, Caplan A. Right-to-try laws: hope, hype, and unintended consequences. Ann. Intern. Med. 163(10), 796-797 (2015).

12. REGROW Act of 2016, S. 2689, 114th Cong. (2016).

13. Turner L, Knoepfler P. Selling stem cells in the USA: assessing the direct-to-consumer industry. Cell Stem Cell 19(2), 154-157 (2016).

14. Young S, Karr A. Deming, data and observational research: a process out of control and needs fixing. Significance 116-120 (2011).

15. Dahabreh IJ, Kent DM. Can the learning health care system be educated with observational data? JAMA 312(2), 129-130 (2014).

16. Lambert H. Accounting for EBM: notions of evidence in medicine. Soc. Sci. Med. 62(11), 2633-2645 (2006).

17. Weisz G. From clinical counting to evidence-based medicine. In: Body Counts: Medical Quantification in Historical and Sociological Perspective. Jorland G, Opinel A, Weisz G (Eds). McGill-Queen's University Press, MO, Canada, 377-393 (2005).

18. Timmermans S, Berg M. The Gold Standard: The Challenge of Evidence-Based Medicine and Standardization in Health Care. Temple University Press, PA, USA (2010).

19. Marks HM. The Progress of Experiment: Science and Therapeutic Reform in the United States, 1900-1990. Cambridge University Press, Cambridge, UK (2000).

20. Begley CG, Ioannidis JPA. Reproducibility in science: improving the standard for basic and preclinical research. Circ. Res. 116(1), $116-126$ (2015).

21. Rosemann A, Chaisinthop N. The pluralization of the international: resistance and alter-standardization in regenerative stem cell medicine. Soc. Stud. Sci. 46(1), 112-139 (2016).

22. Hudis CA. Big data: are large prospective randomized trials obsolete in the future? Breast 24, S15-S18 (2015).

23. Löwy I. The experimental body. In: Companion to Medicine in the Twentieth Century. Cooter R (Ed.). Routledge, London, UK, $435-450$ (2003).

24. Epstein S. Impure science: AIDS, activism, and the politics of knowledge. University of California Press, CA, USA (1996) 
25. Hogle LF. Intersections of technological and regulatory zones in regenerative medicine. In: Intersections: Global Perspectives on Stem Cell Technologies. Bharadwaj A (Ed.). Palgrave Macmillan, Basingstoke, UK (2017).

26. US FDA/Office of the Chief Scientist. Advancing regulatory science for public health (2010). www.fda.gov/ScienceResearch/SpecialTopics/RegulatoryScience/ucm228131.htm

27. London AJ, Kimmelman J, Emborg ME. Beyond access vs. protection in trials of innovative therapies. Science 328(5980), 829-830 (2010).

28. 21 C.F.R. $\S$ 600.3. Electronic Code of Federal Regulation. (2016). www.ecfr.gov/cgi-bin/text-idx?SID=f48cd5c3de5665ef29b7300942c401a9\&mc=true\&tpl=/ecfrbrowse/Title21/21cfr600_main_02.tpl

29. Marks PW, Witten CM, Califf RM. Clarifying stem-cell therapy's benefits and risks. N. Engl. J. Med. 376(11), 1007-1009 (2017).

30. Epstein S. Inclusion: The Politics of Difference in Medical Research. University of Chicago Press, IL, USA (1996). 